\title{
Solvability of the functional integro-differential equation with self-reference and state-dependence
}

\author{
A. M. A. El-Sayed ${ }^{\mathrm{a}}$, Reda Gamal Aahmed ${ }^{\mathrm{b}, *}$ \\ ${ }^{a}$ Faculty of Science, Alexandria University, Egypt. \\ ${ }^{b}$ Faculty of Science, Al-Azhar University, Cairo, Egypt.
}

\begin{abstract}
The existence of solutions of a functional integro-differential equation with self-reference and state-dependence will be studied. The continuous dependence of the solution on the delay $\phi(t)$, the functional $g$ and initial data $x_{0}$ will be proved.
\end{abstract}

Keywords: Functional equations, existence of solutions, continuous dependence, state-dependence, self-reference.

2010 MSC: 34A12, 34A30, 34D20.

(C)2020 All rights reserved.

\section{Introduction}

The differential and integral equations with deviating arguments that appear in recent literature, the deviation of the argument usually involves only the time itself, see [1,2]. However, another case, in which the deviating arguments depend on both the state variable $x$ and the time $t$, is of importance in theory and practice. Several papers have appeared recently that are devoted to such kind of differential equations, see for example [3-6, 9-12] and the references cited therein.

The first papers studying this class of functional equations with self-reference, Eder [5], studied the existence of the unique solution for the differential equation

$$
\chi^{\prime}(t)=x(x(t)), \quad x(0)=x_{0}, \quad t \in B \subset \mathbb{R} .
$$

Wang [11], studied the strong solution and maximal strong solution for the equation

$$
x^{\prime}(t)=f(x(x(t)))
$$

where $f: \mathbb{R} \rightarrow \mathbb{R}$ is continuous and monotone, and $f(0)=0$.

Feckan [6], studied the existence solution

$$
x^{\prime}(t)=f(x(x(t))), \quad x(0)=0, \quad \text { where } f \in C^{1}(\mathbb{R}, \mathbb{R}) .
$$

\footnotetext{
*Corresponding author

Email addresses: amasayed@alexu.edu.eg (A. M. A. El-Sayed), redagamal@azhar.edu.eg (Reda Gamal Aahmed)

doi: $10.22436 /$ jnsa.013.01.01
}

Received: 2019-03-08 Revised: 2019-07-17 Accepted: 2019-07-23 
Buica, studied the existence and continuous dependence of solutions, on $x_{0}$ of the functional-differential equation [4]

$$
x^{\prime}(t)=f(t, x(x(t))), \quad x\left(t_{0}\right)=x_{0}, \quad t \in[a, b],
$$

where $\mathrm{t}_{0}, \mathrm{x}_{0} \in[\mathrm{a}, \mathrm{b}]$ and $\mathrm{f} \in \mathrm{C}([\mathrm{a}, \mathrm{b}],[\mathrm{a}, \mathrm{b}])$.

Stanek [9], studied global properties of decreasing solution of the equation

$$
x^{\prime}(t)=x(x(t))+x(t) .
$$

Stanek [10], studied global properties of solution of functional differential equation

$$
x(t) x^{\prime}(t)=k x(x(t)), \quad 0<|k|<1 .
$$

Here we are concerning with the initial value problem of functional integro-differential equation of selfreference $(\phi(t)=t)$ and state-dependence $(\phi(t) \leqslant t)$

$$
\frac{d x}{d t}=f\left(t, x\left(\int_{0}^{\phi(t)} g(s, x(s)) d s\right)\right), \quad t \in(0, T],
$$

with the condition

$$
x(0)=x_{0},
$$

The existence of solutions $x \in C[0, T]$ will be studied under assumptions on the functional $f$ and $g$. The uniqueness of the solution can be deduce, when $f$ and $g$ are Lipschitz continuous. The continuous dependence of the solution on the delay $\phi(t)$, the functional $g$ and initial data $x_{0}$ will be proved.

\section{Integral representation} tions:

Consider the initial value problem of functional integro-differential equation (1.1) with the assump-

(1) $f:[0, T] \times \mathbb{R} \rightarrow \mathbb{R}$ satisfies Caratheodory condition, i.e., $f$ is measurable in $t$ for any $x \in \mathbb{R}$ and continuous in $x$ for almost all $t \in[0, T]$. There exist a function $c(t)$ that is measurable bounded and there is a positive constant $b>0$, such that

$$
|f(t, x)| \leqslant c(t)+b|x|, \quad|c(t)| \leqslant M ;
$$

(2) $g:[0, T] \times \mathbb{R} \rightarrow \mathbb{R}^{+}$satisfies Caratheodory condition, i.e., $g$ is measurable in $t$ for any $x \in \mathbb{R}$ and continuous in $x$ for almost all $t \in[0, T]$, such that $|g(t, x)| \leqslant 1,(t, x) \in[0, T] \times \mathbb{R}$;

(3) $\phi:[0, T] \rightarrow[0, T]$ is continuous;

(4) $\mathrm{bT}<1$.

Definition 2.1. By a solution of the integral equation (2.1) we mean a function $x \in C[0,1]$ that satisfies (2.1).

Lemma 2.2. Let the solution of the initial value problem (1.1)-(1.2) exists, then it can be represented by the integral equation

$$
x(t)=x_{0}+\int_{0}^{t} f\left(s, x\left(\int_{0}^{\phi(s)} g(\theta, x(\theta)) d \theta\right)\right) d s, \quad t \in[0, T] .
$$

Proof. Let $x$ be a solution of the initial value problem of functional differential equation (1.1)-(1.2). Integrating both sides of (1.1) we obtain

$$
x(t)=x_{0}+\int_{0}^{t} f\left(s, x\left(\int_{0}^{\phi(s)} g(\theta, x(\theta)) d \theta\right)\right) d s, \quad t \in[0, T] .
$$




\section{Existence of solution}

Theorem 3.1. Let the assumptions (1)-(4) be satisfied, then the integral equation (2.1) has at least one solution.

Proof. Define the operator $A$ associated with the integral equation (2.1) by

$$
A x(t)=x_{0}+\int_{0}^{t} f\left(s, x\left(\int_{0}^{\phi(s)} g(\theta, x(\theta)) d \theta\right)\right) d s .
$$

Let $S_{r}=\{x \in \mathbb{R}:|x(t)-x(s)| \leqslant L(t-s), L>0,\|x\| \leqslant r, \forall t, s \in[0, T]\}$, where $r=L T+\left|x_{0}\right|$ and $L=\frac{M+b\left|x_{0}\right|}{1-b T}$. Then we have, for $x \in S_{r}$,

$$
\begin{aligned}
|A x(t)| & =\left|x_{0}+\int_{0}^{t} f\left(s, x\left(\int_{0}^{\phi(s)} g(\theta, x(\theta)) d \theta\right)\right) d s\right| \\
& \leqslant\left|x_{0}\right|+\int_{0}^{t}\left|f\left(s, x\left(\int_{0}^{\phi(s)} g(\theta, x(\theta)) d \theta\right)\right)\right| d s \\
& \leqslant\left|x_{0}\right|+\int_{0}^{t}\left(c(s)+b\left|x\left(\int_{0}^{\phi(s)} g(\theta, x(\theta)) d \theta\right)-x(0)\right|+b|x(0)|\right) d s \\
& \leqslant\left|x_{0}\right|+\int_{0}^{t}\left(c(s)+b L \int_{0}^{\phi(s)}|g(\theta, x(\theta))| d \theta+b|x(0)|\right) d s \\
& \leqslant\left|x_{0}\right|+T M+b L T^{2}+b T\left|x_{0}\right| \leqslant\left|x_{0}\right|+L T .
\end{aligned}
$$

Now, let $t_{1}, t_{2} \in(0, T]$ such that $\left|t_{2}-t_{1}\right|<\delta$, then

$$
\begin{aligned}
\left|A x\left(t_{2}\right)-A x\left(t_{1}\right)\right| & =\left|x_{0}+\int_{0}^{t_{2}} f\left(s, x\left(\int_{0}^{\phi(s)} g(\theta, x(\theta)) d \theta\right)\right) d s-x_{0}-\int_{0}^{t_{1}} f\left(s, x\left(\int_{0}^{\phi(s)} g(\theta, x(\theta)) d \theta\right)\right) d s\right| \\
& \leqslant \int_{t_{1}}^{t_{2}}\left|f\left(s, x\left(\int_{0}^{\phi(s)} g(\theta, x(\theta)) d \theta\right)\right)\right| d s \\
& \leqslant \int_{t_{1}}^{t_{2}}\left(c(s)+b\left|x\left(\int_{0}^{\phi(s)} g(\theta, x(\theta)) d \theta\right)-x(0)\right|+b\left|x_{0}\right|\right) d s \\
& \leqslant \int_{t_{1}}^{t_{2}}\left(c(s)+b L \int_{0}^{\phi(s)}|g(\theta, x(\theta))| d \theta+b\left|x_{0}\right|\right) d s \\
& \leqslant\left(t_{2}-t_{1}\right) M+\left(t_{2}-t_{1}\right) b L T+\left(t_{2}-t_{1}\right) b\left|x_{0}\right|=\left(t_{2}-t_{1}\right)\left(M+b L T+b\left|x_{0}\right|\right)=\left(t_{2}-t_{1}\right) L .
\end{aligned}
$$

This proves that $A: S_{r} \rightarrow S_{r}$ and the class of functions $\{A x\}$ is uniformly bounded And equi-continuous in $S_{r}$.

Let $x_{n} \in S_{r}, x_{n} \rightarrow x(n \rightarrow \infty)$, then from the continuity of the functions $f$ and $g$, we obtain $f\left(t, x_{n}(t)\right) \rightarrow$ $f(t, x(t))$ and $g\left(t, x_{n}(t)\right) \rightarrow g(t, x(t))$ as $n \rightarrow \infty$. Also

$$
\lim _{n \rightarrow \infty} A x_{n}(t)=\lim _{n \rightarrow \infty}\left[x_{0}+\int_{0}^{t} f\left(s, x_{n}\left(\int_{0}^{\phi(s)} g\left(\theta, x_{n}(\theta)\right) d \theta\right)\right) d s\right]
$$

Now

$$
\begin{aligned}
\left|x_{\mathfrak{n}}\left(\int_{0}^{\phi(s)} g\left(\theta, x_{\mathfrak{n}}(\theta)\right) d \theta\right)-x\left(\int_{0}^{\phi(s)} g(\theta, x(\theta)) d \theta\right)\right| \leqslant & \left|x_{\mathfrak{n}}\left(\int_{0}^{\phi(s)} g\left(\theta, x_{\mathfrak{n}}(\theta)\right) d \theta\right)-x_{\mathfrak{n}}\left(\int_{0}^{\phi(s)} g(\theta, x(\theta)) d \theta\right)\right| \\
& +\left|x_{\mathfrak{n}}\left(\int_{0}^{\phi(s)} g(\theta, x(\theta)) d \theta\right)-x\left(\int_{0}^{\phi(s)} g(\theta, x(\theta)) d \theta\right)\right| \\
& \leqslant L \int_{0}^{\phi(s)}\left|g\left(\theta, x_{n}(\theta)\right)-g(\theta, x(\theta))\right| d \theta+\frac{\epsilon}{2} \leqslant \frac{\epsilon}{2}+\frac{\epsilon}{2}=\epsilon .
\end{aligned}
$$


Using assumptions (1)-(2) and Lebesgue dominated convergence theorem [8] we obtain

$$
\lim _{n \rightarrow \infty} A x_{n}(t)=\left[x_{0}+\int_{0}^{t} f\left(s, \lim _{n \rightarrow \infty} x_{n}\left(\int_{0}^{\phi(s)} g\left(\theta, x_{n}(\theta)\right) d \theta\right)\right) d s\right]=A x(t) .
$$

Then $A x_{n} \rightarrow A x$ as $n \rightarrow \infty$. This mean that the operator $A$ is continuous.

Hence by Schauder fixed point Theorem [7] there exist at least one solution $x \in C[0, T]$ of the integral equation (2.1).

Also, differentiating (2.1), we obtain

$$
\frac{d x}{d t}=\frac{d}{d t}\left\{x_{0}+\int_{0}^{t} f\left(s, x\left(\int_{0}^{\phi(s)} g(\theta, x(\theta)) d \theta\right)\right) d s\right\}=f\left(s, x\left(\int_{0}^{\phi(s)} g(\theta, x(\theta)) d \theta\right)\right) .
$$

And at $t=0$ we get

$$
x(0)=x_{0},
$$

then there exist at least one solution $x \in C[0, T]$ of the initial value problem of functional differential equation (1.1)-(1.2).

\section{Uniqueness of the solution}

Let $f$ and $g$ satisfy the following assumptions:

(5) $f:[0, T] \times \mathbb{R} \rightarrow \mathbb{R}$ is measurable in $t$ for any $x \in \mathbb{R}$ and satisfies the Lipschitz condition

$$
|f(t, x)-f(t, u)| \leqslant b|x-u| ;
$$

(6) $g:[0, T] \times \mathbb{R} \rightarrow \mathbb{R}$ is measurable in $t$ for any $x \in \mathbb{R}$ and satisfies the Lipschitz condition

$$
|g(t, x)-g(t, u)| \leqslant b_{1}|x-u|
$$

(7) $\phi:[0, T] \rightarrow[0, T]$ is continuous;

(8) $\left(\mathrm{bb}_{1} \mathrm{LT}^{2}+\mathrm{bT}\right)<1$.

Theorem 4.1. Let the assumptions (5)-(8) be satisfied, then the solution of the integral equation (2.1) is unique.

Proof. From assumption (5) we have $f$ is measurable in $t$ for any $x, y \in \mathbb{R}$ and satisfies the Lipschitz condition, then it is continuous in $x \in \mathbb{R} \forall \mathrm{t} \in[0, \mathrm{~T}]$, and

$$
|f(t, x)| \leqslant b|x|+|f(t, 0)| .
$$

Then condition (1) is satisfied. Also by the same way we can show that assumption (2) satisfied by assumption (5). Now, from Theorem (3.1) the solution of the integral equation (2.1) exists.

Let $x, y$ be two the solutions of (2.1), then

$$
\begin{aligned}
|x(t)-y(t)| & =\left|x_{0}+\int_{0}^{t} f\left(s, x\left(\int_{0}^{\phi(s)} g(\theta, x(\theta)) d \theta\right)\right) d s-x_{0}-\int_{0}^{t} f\left(s, y\left(\int_{0}^{\phi(s)} g(\theta, y(\theta)) d \theta\right)\right) d s\right| \\
& \leqslant \int_{0}^{t}\left|f\left(s, x\left(\int_{0}^{\phi(s)} g(\theta, x(\theta)) d \theta\right)\right)-f\left(s, y\left(\int_{0}^{\phi(s)} g(\theta, y(\theta)) d \theta\right)\right)\right| d s \\
& \leqslant b \int_{0}^{t}\left|x\left(\int_{0}^{\phi(s)} g(\theta, x(\theta)) d \theta\right)-y\left(\int_{0}^{\phi(s)} g(\theta, y(\theta)) d \theta\right)\right| d s \\
& \leqslant b \int_{0}^{t}\left|x\left(\int_{0}^{\phi(s)} g(\theta, x(\theta)) d \theta\right)-x\left(\int_{0}^{\phi(s)} g(\theta, y(\theta)) d \theta\right)\right| d s
\end{aligned}
$$




$$
\begin{aligned}
& \quad+b \int_{0}^{t}\left|x\left(\int_{0}^{\phi(s)} g(\theta, y(\theta)) d \theta\right)-y\left(\int_{0}^{\phi(s)} g(\theta, y(\theta)) d \theta\right)\right| d s \\
& \leqslant b L \int_{0}^{t} \int_{0}^{\phi(s)}|g(\theta, x(\theta))-g(\theta, y(\theta))| d \theta d s+b T\|x-y\| \\
& \leqslant b b_{1} L \int_{0}^{t} \int_{0}^{\phi(s)}|x(\theta)-y(\theta)| d \theta d s+b T\|x-y\| \\
& \leqslant b b_{1} L^{2}\|x-y\|+b T\|x-y\|=\left(b_{1} \mathrm{LT}^{2}+b T\right)\|x-y\| .
\end{aligned}
$$

Hence

$$
\left(1-\left(b b_{1} L^{2}+b T\right)\right)\|x-y\| \leqslant 0 .
$$

Since $\left(b b_{1} L T^{2}+b T\right)<1$, then $x(t)=y(t)$ and the solution of the integral equation (2.1) is unique.

\section{Continuous dependence}

\subsection{Continuous dependence on initial data $x_{0}$}

Definition 5.1. The solution $x \in \mathrm{C}[0, \mathrm{~T}]$ of the integral equation (2.1) depends continuously on initial data $x_{0}$, if

$$
\forall \epsilon>0, \quad \exists \quad \delta(\epsilon) \text { s.t }\left|x_{0}-x_{0}^{*}\right|<\delta \Rightarrow\left\|x-x^{*}\right\|<\epsilon,
$$

where $x^{*}$ is the solution of the integral equation

$$
x^{*}(t)=x_{0}^{*}+\int_{0}^{t} f\left(t, x^{*}\left(\int_{0}^{\phi(s)} g\left(\theta, x^{*}(\theta)\right) d \theta\right)\right) d s, \quad t \in[0, T] .
$$

Theorem 5.2. Let the assumptions of Theorem 4.1 be satisfied, then the solution of the integral equation (2.1) depends continuously on initial data $x_{0}$.

Proof. Let $x$ and $x^{*}$ be two solutions of the integral equation (2.1) and (5.1), respectively, then

$$
\begin{aligned}
\left|x(t)-x^{*}(t)\right|= & \left|x_{0}+\int_{0}^{t} f\left(t, x\left(\int_{0}^{\phi(s)} g(\theta, x(\theta)) d \theta\right)\right) d s-x_{0}^{*}-\int_{0}^{t} f\left(t, x^{*}\left(\int_{0}^{\phi(s)} g\left(\theta, x^{*}(\theta)\right) d \theta\right)\right) d s\right| \\
\leqslant & \left|x_{0}-x_{0}^{*}\right|+\int_{0}^{t}\left|f\left(t, x\left(\int_{0}^{\phi(s)} g(\theta, x(\theta)) d \theta\right)\right)-f\left(t, x^{*}\left(\int_{0}^{\phi(s)} g\left(\theta, x^{*}(\theta)\right) d \theta\right)\right)\right| d s \\
\leqslant & \delta+b \int_{0}^{t}\left|x\left(\int_{0}^{\phi(s)} g(\theta, x(\theta)) d \theta\right)-x^{*}\left(\int_{0}^{\phi(s)} g\left(\theta, x^{*}(\theta) d \theta\right)\right)\right| d s \\
\leqslant & \delta+b \int_{0}^{t}\left|x\left(\int_{0}^{\phi(s)} g(\theta, x(\theta)) d \theta\right)-x\left(\int_{0}^{\phi(s)} g\left(\theta, x^{*}(\theta) d \theta\right)\right)\right| d s \\
& +b \int_{0}^{t}\left|x\left(\int_{0}^{\phi(s)} g\left(\theta, x^{*}(\theta) d \theta\right)\right)-x^{*}\left(\int_{0}^{\phi(s)} g\left(\theta, x^{*}(\theta) d \theta\right)\right)\right| d s \\
\leqslant & \delta+b L \int_{0}^{t}\left|\int_{0}^{\phi(s)} g(\theta, x(\theta)) d \theta-\int_{0}^{\phi(s)} g\left(\theta, x^{*}(\theta)\right) d \theta\right| d s+b \int_{0}^{t}\left\|x-x^{*}\right\| d s \\
\leqslant & \delta+b L \int_{0}^{t} \int_{0}^{\phi(s)}\left|g(\theta, x(\theta))-g\left(\theta, x^{*}(\theta)\right)\right| d \theta d s+b \int_{0}^{t}\left\|x-x^{*}\right\| d s \\
\leqslant & \delta+b L b_{1} \int_{0}^{t} \int_{0}^{\phi(s)}\left|x(\theta)-x^{*}(\theta)\right| d \theta d s+b \int_{0}^{t}\left\|x-x^{*}\right\| d s \\
\leqslant & \delta+b L b_{1} T^{2}\left\|x-x^{*}\right\|+b T\left\|x-x^{*}\right\| .
\end{aligned}
$$

Hence

$$
\left\|x-x^{*}\right\| \leqslant \frac{\delta}{1-\left(b b_{1} T^{2}+b T\right)}=\epsilon .
$$

This mean that the solution of the integral equation (2.1) depends continuously on $x_{0}$. The proof is completed. 
5.2. Continuous dependence on the delay $\phi(t)$

Definition 5.3. The solution $x \in \mathrm{C}[0, \mathrm{~T}]$ of the integral equation (2.1) depends continuously on the delay $\phi(t)$, if

$$
\forall \epsilon>0, \quad \exists \delta(\epsilon) \quad \text { s.t }\left|\phi(t)-\phi^{*}(t)\right|<\delta \Rightarrow\left\|x-x^{*}\right\|<\epsilon,
$$

where $x^{*}$ is the solution of the integral equation

$$
x^{*}(t)=x_{0}+\int_{0}^{t} f\left(t, x^{*}\left(\int_{0}^{\phi^{*}(t)} g\left(\theta, x^{*}(\theta)\right) d \theta\right)\right) d s, \quad t \in[0, T] .
$$

Theorem 5.4. Let the assumptions of Theorem 4.1 be satisfied, then the solution of the integral equation (2.1) depends continuously on the delay $\phi(t)$.

Proof. Let $x$ and $x^{*}$ be two solutions of the integral equation (2.1) and (5.2), respectively, then

$$
\begin{aligned}
\left|x(t)-x^{*}(t)\right|= & \left|x_{0}+\int_{0}^{t} f\left(t, x\left(\int_{0}^{\phi(s)} g(\theta, x(\theta)) d \theta\right)\right) d s-x_{0}-\int_{0}^{t} f\left(t, x^{*}\left(\int_{0}^{\phi^{*}(s)} g\left(\theta, x^{*}(\theta)\right) d \theta\right)\right) d s\right| \\
\leqslant & \int_{0}^{t}\left|f\left(t, x\left(\int_{0}^{\phi(s)} g(\theta, x(\theta)) d \theta\right)\right)-f\left(t, x^{*}\left(\int_{0}^{\phi^{*}(s)} g\left(\theta, x^{*}(\theta)\right) d \theta\right)\right)\right| d s \\
\leqslant & b \int_{0}^{t}\left|x\left(\int_{0}^{\phi(s)} g(\theta, x(\theta)) d \theta\right)-x^{*}\left(\int_{0}^{\phi^{*}(s)} g\left(\theta, x^{*}(\theta) d \theta\right)\right)\right| d s \\
\leqslant & b \int_{0}^{t}\left|x\left(\int_{0}^{\phi(s)} g(\theta, x(\theta)) d \theta\right)-x\left(\int_{0}^{\phi^{*}(s)} g\left(\theta, x^{*}(\theta) d \theta\right)\right)\right| d s \\
& +b \int_{0}^{t}\left|x\left(\int_{0}^{\phi^{*}(s)} g\left(\theta, x^{*}(\theta) d \theta\right)\right)-x^{*}\left(\int_{0}^{\phi^{*}(s)} g\left(\theta, x^{*}(\theta) d \theta\right)\right)\right| d s \\
\leqslant & b L \int_{0}^{t}\left|\int_{0}^{\phi(s)} g(\theta, x(\theta)) d \theta-\int_{0}^{\phi^{*}(s)} g\left(\theta, x^{*}(\theta)\right) d \theta\right| d s+b \int_{0}^{t}\left\|x-x^{*}\right\| d s \\
\leqslant & b L \int_{0}^{t} \mid \int_{0}^{\phi(s)} g(\theta, x(\theta)) d \theta-\int_{0}^{\phi^{*}(s)} g(\theta, x(\theta)) d \theta \\
& +\int_{0}^{\phi^{*}(s)} g(\theta, x(\theta)) d \theta-\int_{0}^{\phi^{*}(s)} g\left(\theta, x^{*}(\theta)\right) d \theta \mid d s+b \int_{0}^{t}\left\|x-x^{*}\right\| d s \\
\leqslant & b L \int_{0}^{t}\left(\int_{0}^{\phi^{*}(s)}\left|g(\theta, x(\theta))-g\left(\theta, x^{*}(\theta)\right)\right| d \theta+\int_{\phi^{*}(s)}^{\phi(s)}|g(\theta, x(\theta))| d \theta\right) d s+b \int_{0}^{t}\left\|x-x^{*}\right\| d s \\
\leqslant & b L \int_{0}^{t}\left(b_{1} \int_{0}^{\phi^{*}(s)}\left|x(\theta)-x^{*}(\theta) d \theta\right|+\int_{\phi^{*}(s)}^{\phi(s)}|g(\theta, x(\theta)) d \theta|\right) d s+b \int_{0}^{t}\left\|x-x^{*}\right\| d s \\
\leqslant & b L b_{1} T^{2}\left\|x-x^{*}\right\|+b L T \delta+b T\left\|x-x^{*}\right\| .
\end{aligned}
$$

Hence

$$
\left\|x-x^{*}\right\| \leqslant \frac{b L T \delta}{1-\left(b b_{1} T^{2}+b T\right)}=\epsilon .
$$

This mean that the solution of the integral equation (2.1) depends continuously on delay $\phi(t)$. The proof is completed.

5.3. Continuous dependence on the functional $\mathrm{g}$

Definition 5.5. The solution $x \in C[0, T]$ of the integral equation (2.1) depends continuously on the functional $\mathrm{g}$, if

$$
\forall \epsilon>0, \quad \exists \delta(\epsilon) \quad \text { s.t }\left|g-g^{*}\right|<\delta \Rightarrow\left\|x-x^{*}\right\|<\epsilon,
$$


where $x^{*}$ is the solution of the integral equation

$$
x^{*}(t)=x_{0}+\int_{0}^{t} f\left(t, x^{*}\left(\int_{0}^{\phi(s)} g^{*}\left(\theta, x^{*}(\theta)\right) d \theta\right)\right) d s, \quad t \in[0, T] .
$$

Theorem 5.6. Let the assumptions of Theorem 4.1 be satisfied, then the solution of the integral equation (2.1) depends continuously on the functional $\mathrm{g}$.

Proof. Let $x$ and $x^{*}$ be two solutions of the integral equation (2.1) and (5.3), respectively, then

$$
\begin{aligned}
\left|x(t)-x^{*}(t)\right|= & \left|x_{0}+\int_{0}^{t} f\left(t, x\left(\int_{0}^{\phi(s)} g(\theta, x(\theta)) d \theta\right)\right) d s-x_{0}-\int_{0}^{t} f\left(t, x^{*}\left(\int_{0}^{\phi(s)} g^{*}\left(\theta, x^{*}(\theta)\right) d \theta\right)\right) d s\right| \\
\leqslant & \int_{0}^{t}\left|f\left(t, x\left(\int_{0}^{\phi(s)} g(\theta, x(\theta)) d \theta\right)\right)-f\left(t, x^{*}\left(\int_{0}^{\phi(s)} g^{*}\left(\theta, x^{*}(\theta)\right) d \theta\right)\right)\right| d s \\
\leqslant & b \int_{0}^{t}\left|x\left(\int_{0}^{\phi(s)} g(\theta, x(\theta)) d \theta\right)-x^{*}\left(\int_{0}^{\phi(s)} g^{*}\left(\theta, x^{*}(\theta) d \theta\right)\right)\right| d s \\
\leqslant & b \int_{0}^{t}\left|x\left(\int_{0}^{\phi(s)} g(\theta, x(\theta)) d \theta\right)-x\left(\int_{0}^{\phi(s)} g^{*}\left(\theta, x^{*}(\theta) d \theta\right)\right)\right| d s \\
& +b \int_{0}^{t}\left|x\left(\int_{0}^{\phi(s)} g^{*}\left(\theta, x^{*}(\theta) d \theta\right)\right)-x^{*}\left(\int_{0}^{\phi(s)} g^{*}\left(\theta, x^{*}(\theta) d \theta\right)\right)\right| d s \\
\leqslant & b L \int_{0}^{t} \int_{0}^{\phi(s)}\left|g(\theta, x(\theta))-g^{*}\left(\theta, x^{*}(\theta)\right)\right| d \theta d s+b \int_{0}^{t}\left\|x-x^{*}\right\| d s \\
\leqslant & b L \int_{0}^{t} \int_{0}^{\phi(s)}\left[\left|g(\theta, x(\theta))-g\left(\theta, x^{*}(\theta)\right)\right|+\left|g\left(\theta, x^{*}(\theta)\right)-g^{*}\left(\theta, x^{*}(\theta)\right)\right|\right] d \theta d s+b \int_{0}^{t}\left\|x-x^{*}\right\| d s \\
\leqslant & b L b_{1} \int_{0}^{t} \int_{0}^{\phi(s)}\left|x(\theta)-x^{*}(\theta)\right| d \theta d s+b L T^{2} \delta+b \int_{0}^{t}\left\|x-x^{*}\right\| d s \\
\leqslant & b L b_{1} T^{2}\left\|x-x^{*}\right\|+b T\left\|x-x^{*}\right\|+b L T^{2} \delta .
\end{aligned}
$$

Hence

$$
\left\|x-x^{*}\right\| \leqslant \frac{b L T^{2} \delta}{\left[1-\left(b L b_{1} T^{2}+b T\right)\right]}=\epsilon
$$

This mean that the solution of the integral equation (2.1) depends continuously on the functional $\mathrm{g}$. The proof is completed.

\subsection{Examples}

In this section we offer some examples to illustrate our results.

Example 5.1. Consider the following nonlinear differential equation

$$
\frac{d x}{d t}=\frac{t+2}{t+5}+\frac{1}{4} x\left(\int_{0}^{\alpha t^{\beta}} \frac{\sin ^{2}(x(s))}{s+e^{|x(s)|}} d s\right), \quad \beta \in[1, B], \alpha, t \in(0,1]
$$

with initial condition

$$
x(0)=0
$$

Set

$$
f\left(t, x\left(\int_{0}^{\phi(t)} g(s, x(s)) d s\right)\right)=\frac{t+2}{t+5}+\frac{1}{4} x\left(\int_{0}^{\alpha t^{\beta}} \frac{\cos ^{2}(x(s))}{s+e^{|x(s)|}} d s\right) .
$$


Then

$$
\left|f\left(t, x\left(\int_{0}^{\phi(t)} g(s, x(s)) d s\right)\right)\right| \leqslant \frac{t+2}{t+5}+\frac{1}{4}(|x|),
$$

and also

$$
|g(s, x(s))| \leqslant 1, \quad \alpha t^{\beta} \leqslant t .
$$

It is clear that the assumptions (1)-(4) of Theorem 3.1 are satisfied with $|c(t)|=\left|\frac{t+2}{t+5}\right| \leqslant \frac{1}{2}$ that is measurable bounded, $\mathrm{b}=\frac{1}{4}, \mathrm{bT}=\frac{1}{4}<1$, and $\mathrm{L}=\frac{\frac{1}{2}}{1-\frac{1}{4}}=\frac{2}{3}$. Therefore, by applying to Theorem 3.1, the given initial value problem (5.4)-(5.5) has a continuous solution.

Example 5.2. Consider the following nonlinear differential equation

$$
\frac{\mathrm{d} x}{\mathrm{dt}}=\frac{1}{5}(\mathrm{t}+1)+\frac{x\left(\int_{0}^{\beta \mathrm{t}} \frac{\mathrm{s}}{\cos ^{2}(s)+e^{|x(s)|}}\right)}{\sqrt{\mathrm{t}+4}}, \quad \beta, \mathrm{t} \in(0,1],
$$

with initial condition

$$
x(0)=0
$$

Set

$$
f\left(t, x\left(\int_{0}^{\phi(t)} g(s, x(s)) d s\right)\right)=\frac{1}{5}(t+1)+\frac{x\left(\int_{0}^{t} \frac{\cos ^{2}(s)}{s+e^{|x(s)|}}\right)}{\sqrt{t+4}}
$$

Then

$$
\left|f\left(t, x\left(\int_{0}^{\phi(t)} g(s, x(s)) d s 0\right)\right)\right| \leqslant \frac{1}{5}(t+1)+\frac{1}{2}|x|,
$$

and also

$$
|g(s, x(s))| \leqslant 1, \quad \beta \leqslant t .
$$

It is clear that the assumptions (1)-(4) of Theorem 3.1 are satisfied with $|c(t)|=\left|\frac{1}{5}(t+1)\right| \leqslant \frac{2}{5}$ that is measurable bounded, $\mathrm{b}=\frac{1}{3}, \mathrm{bT}=\frac{1}{2}<1$, and $\mathrm{L}=\frac{\frac{2}{5}}{1-\frac{1}{2}}=\frac{4}{5}$. Therefore, by applying to Theorem 3.1, the given non-local problem (5.6)-(5.7) has a continuous solution.

\section{References}

[1] C. Bacoțiu, Volterra-fredholm nonlinear systems with modified argument via weakly picard operators theory, Carpathian J. Math., 24 (2008), 1-9. 1

[2] M. Benchohra, M. A. Darwish, On unique solvability of quadratic integral equations with linear modification of the argument, Miskolc Math. Notes, 10 (2009), 3-10. 1

[3] V. Berinde, Existence and approximation of solutions of some first order iterative differential equations, Miskolc Math. Notes, 11 (2010), 13-26. 1

[4] A. Buica, Existence and continuous dependence of solutions of some functional-differential equations, Seminar on Fixed Point Theory (Babes-Bolyai Univ., Cluj-Napoca), 3 (1995), 1-14. 1

[5] E. Eder, The functional differential equation $x^{\prime}(t)=x(x(t))$, J. Differential Equations, 54 (1984), 390-400. 1

[6] M. Fečkan, On a certain type of functional differential equations, Math. Slovaca, 43 (1993), 39-43. 1

[7] K. Goebel, W. A. Kirk, Topics in metric fixed point theory, Cambirdge Universty Press, Cambridge, (1990). 3

[8] A. N. Kolomogorov, S. V. Fomin, Inroductory real analysis, Dover Publications, New York, (1975). 3

[9] S. Staněk, Global properties of decreasing solutions of the equation $x^{\prime}(t)=x(x(t))+x(t)$, Funct. Differ. Equ., 4 (1997), 191-213. 1

[10] S. Stanĕk, Globel properties of solutions of the functional differenatial equation $x(t) x^{\prime}(t)=k x(x(t)), 0<|k|<1$, Funct. Differ. Equ., 9 (2004), 527-550. 1

[11] K. Wang, On the equation $x^{\prime}(t)=f(x(x(t)))$, Funkcial. Ekvac., 33 (1990), 405-425. 1

[12] P. P. Zhang, X. B. Gong, Existence of solutions for iterative differential equations, Electron. J. Differential Equations, 2014 (2014), 10 pages. 1 